\title{
FINANÇAS PÚBLICAS E CRESCIMENTO ECONÔMICO NOS MUNICÍPIOS PAULISTAS
}

\author{
Juliana Aline Forlin ${ }^{1}$ \\ Rodrigo Vilela Rodrigues ${ }^{2}$
}

RESUMO: O Desenvolvimento Econômico é uma das mais relevantes funções econômicas do Estado, com o qual o mesmo pode colaborar com gastos que incentivem a geração de renda e o crescimento econômico. Com a Descentralização das atribuições do Estado, a partir da Constituição de 1988, a esfera municipal, cuidando de setores como saúde, educação, com incentivos à produtividade e à qualidade de vida, se tornou ator fundamental nesse processo. Dessa forma, o presente trabalho tem o intuito de avaliar como as Finanças Públicas Municipais se relacionam com o crescimento Econômico, no Estado de São Paulo, centro econômico e financeiro do país. Para tal, baseou-se nas contribuições teóricas dos Modelos Endógenos de Crescimento Econômico (BARRO, 1990) e da Nova Geografia Econômica (KRUGMAN, 1991). Através de estimativas cross-section, em Mínimos Quadrados Ordinários, dos 645 municípios paulistas para o ano de 2000, percebeu-se impacto condizente com a teoria e relevante para as variáveis que indicam a presença do Estado na Economia, tanto do ponto de vista das receitas, quanto das despesas. As relações não convencionais estão devidamente justificadas no texto.

Palavras-chave: São Paulo, setor público, crescimento econômico.

ABSTRACT: The Economic Development is one of the most important economic functions of the State, with which the same it can collaborate with expenses that stimulate the generation of income and the economic growth. With the Decentralization of the attributions of the State, from the Constitution of 1988, the municipal sphere, taking care of sectors as health, education, with incentives to the productivity and the quality of life, if became basic actor in this process. Of this form, the present work has intention to evaluate as the City Public Finances if they relate with the Economic growth, in the State of São Paulo, economic and financial center of the country. For such, it was based on the theoretical contributions of the Endogenous Models of Economic Growth (BARRO, 1990) and of New Economic Geography (KRUGMAN, 1991). Through estimates cross-section, in Ordinary Least Squares, of the 645 São Paulo cities for the year of 2000, perceived impact analogous with the theory for the variables that indicate the presence of the State in the Economy, as much of the point of view of taxes, how much of the expenditures. The not conventional relations duly are justified in the text.

Key-words: São Paulo, Public Sector, Economic Growth.

\section{INTRODUÇÃO}

\footnotetext{
${ }^{1}$ Estudante de Economia da UFSCar/Campus Sorocaba. Artigo fruto de trabalho de Iniciação Científica. Atualmente é estagiária no Banco Itaú, em São Paulo. E-mail: julianaforlin@ gmail.com.

${ }^{2}$ Professor no curso de Ciências Econômicas da Universidade Federal de São Carlos (UFSCar), em Sorocaba. E-mail: rvilela@ufscar.br.
}

Recebido em: 18/05/2012

Aceito em: 14/08/2012 
O crescimento econômico, mensurado através das variações do Produto Interno Bruto (PIB), é uma das muitas medidas utilizadas como indicador de desenvolvimento econômico. A atuação do governo através de seus gastos é um relevante determinante desse crescimento, fato evidenciado teoricamente no trabalho de Barro (1990) e empiricamente em uma série de outros estudos [ROCHA e GIUBERTI (2005), ASSIS e DIAS (2004) HERRERA e BLANCO (2004), KWEKA e MORRISSEY (2000) e CÂNDIDO Jr. (2001)].

O Estado, no Brasil, teve papel determinante no crescimento econômico do país, principalmente no século XX, especialmente até a década de oitenta. Com relação à atuação do Estado $^{3}$, tem se destacado o fenômeno da descentralização e suas implicações em termos de tamanho do Governo e sua eficiência. Nesse tocante, a investigação sobre as causas do crescimento econômico dos municípios e a influência dos gastos públicos nesse crescimento se faz premente.

A literatura econômica trata o fato, considerando a infra-estrutura local, como determinante do crescimento econômico municipal, com dados acerca de oferta de água encanada e luz elétrica, por exemplo, como em Chagas e Toneto Jr. (2003). Uma das hipóteses sobre a descentralização versa que a mesma, ao aproximar a oferta de bens públicos de sua demanda, torna os gastos públicos mais eficientes.

O poder público municipal é, atualmente, o principal protagonista na área de saúde pública, de educação pré-escolar e fundamental. É ele, também, o responsável por serviços públicos da maior relevância para a saúde ambiental e a qualidade de vida, como a coleta, o tratamento e a disposição de resíduos, o abastecimento de água e o esgotamento sanitário.

Oliveira (2006) afirma que gastos produtivos ajudam a equilibrar a distribuição espacial das atividades, afetando positivamente o crescimento econômico dos municípios. É aparentemente inegável, a importância do governo local para o crescimento dos municípios, sendo assim, a política fiscal local deve ser bem elaborada, para gerar o máximo de benefícios econômicos e sociais.

O Estado de São Paulo é o principal centro econômico e financeiro do país, apresenta o maior e mais completo parque industrial, atuando em diferentes tipos de produção. A concentração dos setores industriais, ligados à produção de bens de alto

\footnotetext{
${ }^{3}$ A relevância do Estado como indutor de desenvolvimento econômico encontra-se como uma das funções do Estado em títulos como Rezende (2007), Musgrave (1959) e Giambiagi (2008).
} 
valor agregado e conteúdo tecnológico, possibilita a comparação da economia paulista à de países mais desenvolvidos que o Brasil (SECRETARIA DE ECONOMIA E PLANEJAMENTO, 2007). Além de destacar-se no setor industrial, São Paulo também é relevante na produção agrícola, com índices de produtividade da terra e do trabalho, comparáveis às economias mais avançadas do mundo.

Sendo responsável por mais de $31 \%$ do PIB do país, com uma população que representa cerca de $22 \%$ da população brasileira, São Paulo legitima seu status de "locomotiva econômica" do Brasil, por possuir atributos como infra-estrutura e mão-deobra qualificada. Além disso, o estado possui o terceiro melhor índice de desenvolvimento humano (IDH) do país.

São Paulo subdivide-se em 645 municípios, 14 regiões administrativas e três regiões metropolitanas. A região metropolitana de São Paulo mantém seu papel de liderança, em termos econômicos e densidade populacional, podendo ser classificada a mais importante área metropolitana da América Latina. Com concentrações menores, porém com grande dinamismo econômico, destacam-se as regiões metropolitanas de Campinas e da Baixada Santista, que juntamente com as regiões administrativas de Sorocaba e São José dos Campos, concentram cerca de $83 \%$ do PIB estadual (SEADE, 2006).

Apesar da concentração, a atividade econômica não se restringe a região metropolitana de São Paulo e regiões vizinhas. Nas demais regiões do estado, prevalece a indústria agroalimentar. No entanto, algumas cidades se destacam em outros setores, como Ribeirão Preto, no setor de instrumentação médico-hospitalar, de precisão e de automação, e São Carlos, importante centro tecnológico.

O bom desempenho econômico do Estado de São Paulo não é um fato recente. O início do sucesso data, na região metropolitana de São Paulo, do fim do século XIX, com o auge da produção cafeeira, passando pela década de 50 com a indústria automobilística até os anos 70, quando a região concentrou grande parte da atividade econômica nacional. Neste sentido, Gonçalves e Resende (1993) ressaltam que um fator fundamental para o crescimento econômico do Estado de São Paulo foi a ação estatal, que realizou grandes investimentos para a formação de mão-de-obra qualificada, criação de infra-estrutura urbana e de transportes.

A partir da década de 80 , com a redemocratização política, intensificou o processo de desconcentração industrial. Tal fato fez com que maiores investimentos fossem destinados para fora da região metropolitana de São Paulo, o que alterou de 
modo significativo a configuração regional (Azzoni, 1993). Com a maior modernização e fortalecimento dos municípios paulistas, alargou-se a base industrial e consolidaramse as vantagens competitivas do Estado.

Atualmente, dos 30 maiores municípios brasileiros em termos econômicos, 12 estão no Estado de São Paulo, de acordo com dados do PIB (Produto Interno Bruto) municipal do ano de 2007 (SEADE, 2010). Em termos de crescimento populacional, as cidades paulistas apresentam taxas médias de crescimento maiores que do restante do país, o que reflete a forte atração que São Paulo exerce, diante dos outros estados da Federação. Tal comportamento pode ser explicado pela concentração das atividades produtivas e sua capacidade de geração de renda.

Em termos de contas públicas, o estado de São Paulo não perde a liderança. Segundo dados do IPEA (2011), São Paulo supera os outros estados brasileiros, quando se trata de finanças públicas. As despesas de capital do estado, ou seja, as despesas destinadas a construção e aquisição de bens de capital atingiram, em 2005, aproximadamente 3 bilhões de reais, sendo que a média brasileira para este ano foi 0,5 bilhões. Já as despesas de custeio, utilizadas para a manutenção dos serviços públicos, demonstra ainda mais a diferença que o estado de São Paulo apresenta em relação aos outros estados. Em 2005, as despesas de custeio do estado de São Paulo atingiram 12,3 bilhões, enquanto os outros estados gastaram em média 1,2 bilhões.

Com relação às contas municipais, os municípios do estado de São Paulo também apresentam um grande diferencial, comparados ao outros municípios brasileiros. As transferências de capital para os municípios do estado de São Paulo atingiram, em média, nos últimos anos, 601 milhões, enquanto a média mais próxima foi 340 milhões, transferidos para os municípios do Rio de Janeiro, nos últimos anos.

Costa et al (2007) afirma que uma boa política fiscal, realizada pela prefeitura, mostra na maioria das vezes resultados positivos ${ }^{4}$. Esta política deve ser eficiente, no âmbito de gerar maior qualidade de vida e possibilitar um padrão mais elevado na produtividade de diversos setores econômicos. Logo, é necessário mostrar para os planejadores econômicos, encarregados das decisões de investimento dos recursos da prefeitura, qual tipo de gasto afeta, de maneira mais contundente, o crescimento dos municípios paulistas.

\footnotetext{
${ }^{4}$ Mais informações a respeito dos impactos da política fiscal local no crescimento econômico municipal em Oliveira (2004), Krugman (1991) e Oliveira et al (2009).
} 
A eficácia e eficiência das políticas dependerão de tamanho e porte populacional; situação geográfica, geopolítica e ambiental; perfil de atividades produtivas; da qualidade política e capacidade administrativa de seus líderes, além do grau de organização da sociedade civil. Desta forma, os investimentos que aumentam a qualificação e a qualidade de vida dos indivíduos, merecem destaque na pauta de discussões sobre crescimento econômico. Sendo assim, fica claro, que o investimento público é relevante, para o fornecimento de infra-estrutura e de bens públicos geradores de externalidades positivas, favorecendo as empresas e o próprio crescimento econômico.

\section{REVISÃO DE LITERATURA}

O papel da política fiscal é bastante debatido, dentro da teoria do crescimento econômico, com muitos trabalhos que relacionam gastos do governo e crescimento do produto de países. Porém, poucos trabalhos fazem isso para cidades [OLIVEIRA (2004); OLIVEIRA (2006); COSTA e LIMA (2008)]. No entanto, o crescimento econômico de cidades sempre esteve ligado, de alguma forma, ao crescimento econômico das nações.

A teoria do crescimento econômico ganhou notoriedade com os trabalhos de Solow (1956) e Swan (1956). Segundo o modelo "Solow-Swan", que assume retornos constantes a escala e retorno decrescente para cada fator de produção, o nível de investimento tem papel primordial na determinação do nível de renda do país. O nível de investimento exigido é aquele que mantém a relação capital-trabalho constante, e o resultado seria o crescimento de estado estacionário. Romer (1986) afirma que tal modelo apresentava falhas em seus pressupostos, como o rendimento decrescente nos insumos e a competição perfeita.

Neste sentido, após muito tempo que a teoria do crescimento econômico ficou estagnada, Romer (1986) e Lucas (1988) contribuíram para sua evolução. Romer (1986), em seu trabalho, distingue retornos privados de retornos sociais do investimento. No modelo de Romer (1986), os retornos a escala privados serão decrescentes e os retornos sociais, que refletem transbordamento de conhecimento ou externalidades, serão crescentes ou constantes. Neste modelo, o pressuposto básico é que o crescimento econômico de longo prazo origina-se nas externalidades positivas, decorrentes da acumulação de conhecimento tecnológico. 
Já o modelo de Lucas (1988), coloca que o declínio da produtividade marginal do capital pode ser compensado pela qualificação do indivíduo. Neste modelo, é o investimento em capital humano que proporciona as externalidades positivas, através de aumentos no nível tecnológico. Lucas (1988) considera o capital humano (soma de habilidades do indivíduo) um fator acumulável, e a fonte primária de crescimento.

Segundo Barro (1990), tais modelos podem gerar crescimento econômico de longo prazo, sem incorporarem em sua análise, as modificações relacionadas aos avanços tecnológicos, ou ao crescimento populacional. Além disso, nos modelos de crescimento endógeno, os retornos do investimento podem ser constantes ou crescentes, devido às externalidades e o efeito transbordamento de conhecimento.

No geral, nos modelos de crescimento endógeno, a tecnologia é a fonte básica do crescimento, sendo a inovação, o resultado da busca pelo poder de monopólio. Desse modo, a taxa de progresso tecnológico é afetada por investimentos em P\&D, recompensados por alguma forma de poder de monopólio. Entretanto, as inovações tecnológicas nos métodos de produção trazem distorções, o que leva a uma taxa de crescimento que não é ótima no sentido de Pareto, uma vez que gera spillovers, ou seja, externalidades.

Desta forma, estes modelos abrem espaço para as implicações de políticas públicas, uma vez que o governo pode afetar o crescimento de longo prazo dos países, através de taxações, fornecimento de infra-estrutura, leis de patente, regulação de mercados, investimentos, entre outros.

Sob esse contexto, a ação do governo de um país ganha notoriedade, uma vez que a taxa de crescimento pode ser influenciada por suas escolhas políticas. Trabalhos empíricos recentes enfatizam o papel do governo no crescimento econômico. Rocha e Giuberti (2005) fazem uma decomposição dos gastos públicos, para determinar a influência de cada tipo de gasto no crescimento econômico do Brasil. Neste trabalho, verifica-se empiricamente que gastos com capital, defesa, educação, transporte e comunicação, influenciam positivamente o crescimento econômico. Enquanto gastos correntes do governo apresentam uma influencia negativa no crescimento econômico.

Outro trabalho empírico relevante, de Assis e Dias (2004), baseando-se na versão do modelo de crescimento endógeno proposto por Barro (1990), e utilizando um modelo do tipo auto-regressivo vetorial (VAR) nas análises econométricas, verificou, empiricamente, a hipótese de que o impacto sobre o crescimento econômico dependerá dos tributos utilizados, para custear esses gastos. 
Barro (1990) construiu um modelo, que inclui o setor público como insumo produtivo, em uma função de produção. Esta teoria tem implicações nas relações entre o tamanho do governo e as taxas de crescimento e poupança. Os resultados são úteis, para que o governo escolha suas ações de modo não aleatório. No modelo de Barro (1990), o crescimento é gerado endogenamente, através da acumulação de capital e gastos do governo, assim como mostra a equação (1):

$$
y=f(k, g)=A \cdot k^{1-\alpha} g^{\alpha},
$$

onde $y, k, g$ são variáveis de produto, estoque de capital privado e gastos do governo, respectivamente, $\alpha$ é a parcela do governo no produto e $A$ representa o nível de tecnologia. Desta forma, utilizaram-se retornos constantes a escala, para os dois insumos, capital e gastos do governo, quando analisados em conjunto. Analisados separadamente, os dois insumos apresentam retornos marginais decrescentes.

Supõe-se que os gastos do governo são financiados por um imposto proporcional a renda, e que não há endividamento do setor público. Portanto, a restrição orçamentária do governo, se expressa pela equação (2):

$$
g=T=\tau \cdot y=\tau \cdot A k^{1-\alpha} g^{\alpha},
$$

onde $T$ e $g$ são, respectivamente, as receitas tributárias e os gastos do governo. A alíquota $\tau$ representa a taxa média do imposto de renda.

O resultado do modelo mostra que a política fiscal apresenta um efeito ambíguo sobre a economia, de modo que, os gastos do governo apresentam efeitos positivos sobre o crescimento econômico, enquanto os impostos apresentam efeito negativo. Assim sendo, se por um lado, a entrada dos gastos públicos na função de produção gera externalidades positivas, sobre o capital privado, por outro lado, maiores gastos implicam a expansão da carga tributária, diminuindo os recursos disponíveis ao setor privado, impactando negativamente o nível de crescimento econômico. Portanto, o governo, para contribuir com o crescimento econômico, deve buscar um tamanho ótimo do setor público em termos de gasto e tributação.

A partir dessas estruturas teóricas, que explicam o crescimento econômico das nações, é razoável supor, que exista uma ligação entre os determinantes de crescimento 
econômico de regiões, e os fatores que promovem o crescimento econômico de países. Porém, a maioria dos trabalhos que estudam o crescimento econômico de países, como o modelo de Barro (1990), desconsidera a possibilidade de haver mobilidade de capitais e mão-de-obra, dificultando a utilização de tais análises em âmbito regional e municipal. Além disso, tais trabalhos não incorporam em suas análises as economias de aglomeração, ou seja, as externalidades geográficas.

Dentro desse aspecto, vários autores vêm abordando a relação entre externalidades espaciais e crescimento das cidades. Von Thünen (1826), com seu modelo da cidade isolada, que discute as decisões de localização baseando-se no preço da terra e custo de transporte, serviu como base para muitos autores da Ciência Regional, como Hoover e Giarratani (1984) e Henderson (1986).

No entanto, segundo Krugman (1991), muitas dessas teorias, que balizaram a ciência regional, apresentam sérias limitações e problemas teóricos consideráveis. Neste sentido, com o intuito de produzir uma teoria consistente, sobre como os agentes se dispersam no espaço, originou-se a Nova Geografia Econômica, sendo seus principais expoentes Paul Krugman, Masahisa Fujita e Anthony Venables (RUIZ, 2004).

A Nova Geografia Econômica (NGE) diferencia-se das outras teorias, por considerar dois aspectos: o espaço, que influencia na localização das atividades; e as distâncias, que trazem efeitos nos custos de transporte de bens e serviços, tornando, assim, uma região mais atrativa que outra. Sob a visão da NGE, existem externalidades positivas, ou forças centrípetas, que levam à aglomeração das atividades, e externalidades negativas, ou forças centrífugas, que levam a dispersão das atividades entre as regiões.

Krugman (1991) foi o precursor desta teoria, com um modelo centro-periferia, baseado na interação de economias de escala, custos de transporte e migrações. No modelo centro-periferia, os consumidores e firmas distribuem-se entre as regiões e arcam com os custos de transporte, para a compra e venda de mercadorias. Desta forma, ambos tentam minimizar os gastos, localizando-se próximos aos demais agentes, ou seja, onde há uma aglomeração de atividades econômicas.

De acordo com a perspectiva da NGE, a aglomeração das atividades eleva a produtividade, o que influencia as taxas de crescimento de renda e emprego. Neste contexto, Glaeser et. al. (1995) desenvolve um modelo para o crescimento de municípios norte-americanos, incorporando ferramentas fornecidas pela NGE, com a 
intenção de quantificar a questão da localização no crescimento econômico dos municípios.

Com isso, a NGE explica o desenvolvimento e o subdesenvolvimento de uma região, de uma forma que deixa os formuladores de políticas econômicas, apenas com um meio de lutar contra essas forças, a política fiscal. O governo, por meio de incentivos fiscais e investimentos, pode atrair atividades econômicas para a região, e, assim, impactar seu crescimento econômico (OLIVEIRA et al, 2009).

A partir daí, alguns trabalhos incorporaram ao modelo de Glaeser et. al. (1995) e Glaeser (2000), o papel da política fiscal. Oliveira (2004); Oliveira (2006) e Oliveira. et al (2009) incorporam, no modelo de econometria espacial de Glaeser, a política fiscal, seguindo contribuições dos modelos de crescimento endógeno, citados no começo da seção. Neste modelo, admite-se a mobilidade do capital e da mão de obra no espaço, sendo assim, o crescimento econômico local independe da taxa de poupança. Os retornos sobre o capital e a utilidade dos trabalhadores são iguais em todas as cidades, estas irão diferir entre si, somente em níveis de produtividade, qualidade de vida, quantidade de terras disponíveis, custos de transporte e política fiscal local (OLIVEIRA et al, 2009).

O produto de cada cidade pode ser representado pela seguinte função do tipo Cobb-Douglas:

$$
Y_{i, t}=A_{i, t} L_{i, t}^{\sigma} S_{i, t}^{Y} G_{i, t}^{\phi}
$$

para $i$ cidades e $t$ anos

onde, $Y$ representa o produto; $A$ é o nível de produtividade da cidade; $G$ são os gastos do governo; $L$ é a mão de obra e $S$, a quantidade de terra utilizada na produção. Os coeficientes $\sigma, \gamma$ e $\varphi$ medem as elasticidades dos fatores de produção em relação ao produto. Por simplificação, assume-se que a quantidade de terra por trabalhador é uma proporção fixa.

Dado que a produtividade total da cidade depende do número de trabalhadores, tem-se que:

$$
A_{i, t}=a_{i, t} L_{i, t}^{a}
$$


Tal fato implica algum tipo de economia de aglomeração e o parâmetro $\alpha$ reflete a importância desta na produção. Assim, podemos reescrever a equação (3) como:

$$
Y_{i, t}=a_{i, t} L_{i, t}^{(\sigma+\gamma+\alpha)} S_{i, t}^{\gamma} G_{i, t}^{\phi}
$$

Partindo do pressuposto que o governo local tributa com uma alíquota $\tau_{i, t}$ do produto e que o mercado de trabalho é competitivo, a remuneração líquida dos trabalhadores é a produtividade marginal de cada trabalhador subtraindo a tributação, dada por:

$$
W_{i, t}=\left(1-\tau_{i, t}\right)(\sigma+\gamma+\alpha) a_{i, t} L_{i, t}^{(\sigma+\gamma+\alpha)} S_{i, t}^{\gamma} G_{i, t}^{\phi}
$$

Admitindo-se que toda a renda gerada pelo trabalho é gasta, a utilidade monetária total dos trabalhadores $\left(U_{i, t}\right)$ é dada pela equação:

$$
U_{i, t}=\frac{W_{i, t} Q_{i, t}}{I_{i, t}}
$$

onde, $W_{i, t}$ é remuneração dos trabalhadores; $Q_{i, t}$ é um índice de qualidade de vida e $I_{i, t}$ um índice de preços. Desta forma, a utilidade monetária relaciona-se negativamente com o aumento do numero de trabalhadores e positivamente com o crescimento da cidade, ou seja, com o ganho de qualidade de vida. Além disso, ao assumir liberdade de migração entre as cidades, asseguram- se utilidades constantes através do espaço em um ponto do tempo.

Assume-se que tal índice é uma função monotonicamente inversa ao tamanho dos municípios:

$$
Q_{i, t}=q_{i, t} L_{i, t}^{-\beta}
$$

onde $\beta>0$. Denota-se que o índice de qualidade de vida engloba o efeito de diversos fatores, inclusive crime, preço dos imóveis e congestionamento.

Já o índice de preços pode ser decomposto da seguinte forma: 


$$
I_{i, t}=\left(P_{t} T_{i, t}\right)^{1-\theta} R_{i, t}^{\theta}
$$

onde, $P_{t}$ representa o preço dos bens de consumo, que é igual em todas as cidades; já os custos de transporte, que é dado por $T_{i, t}$, diferem entre as cidades. $R_{i, t}$, representa o preço da terra e $\theta$ representa a parcela de cada uma dessas variáveis no custo de vida de cada pessoa.

A oferta de terras depende da quantidade de terra disponível na cidade, dado por uma dotação inicial constante $t_{i, t}$. Assim, o equilíbrio entre oferta e demanda por terra determina o preço da terra $R_{i, t}$ :

$$
\left(s_{t}+c_{t}\right) L_{i, t}=t_{i, t} R_{i, t}^{\xi}
$$

onde, $s_{t}$ o consumo de terras para produção por trabalhador e $c_{t}$ o consumo de terras para residências por trabalhador.

Adicionando um tributo $r_{i, t}$, pago ao governo, sobre a propriedade da terra obtemos:

$$
R_{i, t}=\left(1+r_{i, t}\right)\left(s_{t}+c_{t}\right)^{\frac{1}{\xi}} L_{i, t} \frac{1}{\xi} t_{i, t}^{-\left(\frac{1}{\xi}\right)} .
$$

Esta equação mostra que quanto maior a demanda por terras, o número de trabalhadores e a alíquota do tributo, maior o preço da terra. E um aumento de terras disponíveis para construção na cidade reduz o preço da terra.

Esse modelo espacial se distingue dos modelos de crescimento endógeno, uma vez que a tributação age como força centrífuga. Ou seja, reduz a utilidade dos trabalhadores. Já os gastos do governo agem como força centrípeta, afetando positivamente utilidade dos trabalhadores. Nesse sentido, assume-se que o governo possui um orçamento equilibrado dado por:

$$
G_{i, t}=\tau_{i, t} Y_{i, t}+r_{i, t} R_{i, t}
$$

Substituindo (6), (8), (9) e (11) em (7) e calculando os logaritmos obtém-se: 
$\log U_{i, t}=C_{t}+(\sigma+\gamma+\alpha-\beta-\theta \varepsilon-1) \log L_{i, t}+\log \alpha_{i, t}+\log q_{i, t}-(1-\theta) \log T_{i, t}+\log \left(1-\tau_{i, t}\right)$

onde, $\quad \varepsilon=\frac{1}{\xi} \quad$ e $^{C_{t}=\log (\sigma+\gamma+\alpha)+\gamma \log s_{t}-\theta \varepsilon \log \left[\left(s_{\perp}\right]+c_{t}\right)-(1-\theta) \log P_{t}}$. Aqui, se pressupõe a existência de um equilíbrio espacial, em que os trabalhadores obtêm a mesma utilidade em todas as cidades.

A remuneração da mão de obra é obtida substituindo-se (13) em (6) e aplicando os logaritmos. Com algumas manipulações algébricas, tem-se que:

$\log W_{i, t}=C_{t}^{x^{p}}+m_{1}\left[\left(\log a_{i, t}+\phi \log G_{i, t}+\log \left(1-r_{i, t}\right)\right)\right]+m_{\mathbf{z}}\left[\log q_{i, t}-\theta \log \left(1-r_{i, t}\right)-(1-\theta) \log T_{i, t}\right]+$

onde $\quad m_{1}=\frac{\beta+\theta \varepsilon}{1+\beta+\theta \varepsilon-(\sigma+\gamma+\alpha)}, \quad m_{\mathbf{2}}=\frac{\sigma+\gamma+\alpha-1}{1+\beta+\theta \varepsilon-(\sigma+\gamma+\alpha)} \quad \mathrm{e}$ $C_{t}^{x^{p}}=m_{1}\left[\log (\sigma+\gamma+\alpha)+\gamma \log \mathbf{s}_{\mathrm{t}}\right]-\mathbf{m}_{2}\left[\log \mathbf{U}_{\mathbf{t}}+(\mathbf{1}-\boldsymbol{\theta}) \log \mathbf{P}_{\mathbf{t}}+\theta \varepsilon \log \left(\mathrm{s}_{\mathrm{t}}+\mathbf{c}_{\mathrm{t}}\right)\right]$.

A equação (14) evidencia que o crescimento econômico depende de mudanças em níveis de produtividade, qualidade de vida, quantidade de terras disponíveis, custos de transporte e política fiscal local. Portando, as cidades vão apresentar distintas taxas de crescimento econômico, devido a diferenças nessas variáveis.

\section{MODELO ANALÍTICO}

Na aplicação do modelo teórico, considera-se um vetor $\left(x_{i, t}\right)$ das características das cidades no tempo $t$, que determina tanto o crescimento da qualidade de vida em uma determinada cidade, quanto o crescimento de seu nível de produtividade.

$$
\begin{aligned}
& \log \left(\frac{a_{i, t+1}}{a_{i, t}}\right)=X_{i, t}^{s} \Psi+\eta_{i, t} \\
& \log \left(\frac{q_{i, t+1}}{q_{i, t}}\right)=X_{i, t}^{s} \Omega+\mu_{i, t}
\end{aligned}
$$


onde $\eta$ e $\mu$ são os erros com média zero e variância constante e ortogonais em relação ao vetor de características $X_{i, t}$. Considera-se também que os custos de transporte estão relacionados a um vetor de variáveis $Y_{i, t}$ :

$$
\log \left(\frac{T_{i, t+1}}{T_{i, t}}\right)=Y_{i, t}^{*} \Theta+\rho_{i, t}
$$

onde $\Theta$ é um vetor de parâmetros e $\rho$ é o termo de erro.

A política fiscal local depende de um vetor de variáveis $Z_{i, t}$ associadas a um conjunto de parâmetros.

$$
\log \left(\frac{G_{i, t+1}}{G_{i, t}}\right)=Z_{i, t}^{s} \Phi+\pi_{i, t}
$$

A partir da equação (14) do referencial teórico e dos vetores $X_{i, t}^{\prime}, Y_{i, t}{ }^{\prime}$ e $Z_{i, t}^{\prime}$ obtém-se a equação que determina o crescimento econômico da cidade:

$$
\log \left(\frac{W_{i, t+1}}{W_{i, t}}\right)=m_{1}\left[X_{i, t}^{s} \Psi+Z_{i, t}^{s}(\Phi+K)\right]+m_{\mathbf{2}}\left(X_{i, t}^{s} \Omega+Y_{i, t}^{s} \Theta+Z_{i, t}^{s} \Gamma\right)+v_{i, t}
$$

onde $v_{i, t}$ é o termo de erro com média zero, variância constante e não correlacionados com as características da cidade $X_{i, t}^{\prime}, Y_{i, t}^{\prime}$ e $Z_{i, t}^{\prime}$

O quadro abaixo mostra as variáveis escolhidas que representam as características $\left(X_{i, t}^{\prime}, Y_{i, t}^{\prime}\right.$ e $\left.Z_{i, t}^{\prime}\right)$ da cidade.

Quadro 1: Estatísticas Descritivas das variáveis para os 645 municípios paulistas no ano de 2000.

\begin{tabular}{|c|c|c|c|c|c|}
\hline \multicolumn{2}{|c|}{ Forças Centrípetas } & Média & $\underline{\text { Desvio padrão }}$ & $\underline{\text { Mínimo }}$ & $\underline{\text { Máximo }}$ \\
\hline $\begin{array}{c}\text { Variável } \\
\text { Teórica }\end{array}$ & Proxy & & & & \\
\hline $\begin{array}{c}\text { Externalidades } \\
\text { positivas }\end{array}$ & Grau de Urbanização em \% & 80,63 & 15,45 & 21,48 & 100,00 \\
\hline $\begin{array}{c}\text { Média de anos de estudo de } \\
\text { pessoas com mais de 25 anos. }\end{array}$ & 70,43 & 8,64 & 37,68 & 89,46 \\
\hline $\begin{array}{c}\text { Custos de } \\
\text { transporte }\end{array}$ & $\begin{array}{c}\text { Distância do município em relação } \\
\text { à capital do Estado em Km }\end{array}$ & 343,85 & 182,07 & 0 & 763 \\
\hline $\begin{array}{c}\text { Quantidade de } \\
\text { terras }\end{array}$ & $\begin{array}{c}\text { Área do município medida em } \\
\text { Km² }\end{array}$ & 384,82 & 320,05 & 3,64 & 1980,92 \\
\hline
\end{tabular}




\begin{tabular}{|c|c|c|c|c|c|}
\hline $\begin{array}{l}\text { Gastos do } \\
\text { governo }\end{array}$ & $\begin{array}{c}\text { Gastos do governo municipal } \\
\text { excluindo as despesas de capital - } \\
\mathrm{R} \$ \\
\end{array}$ & $3.414 .432,01$ & $22.161 .057,64$ & 0 & $528.422 .842,00$ \\
\hline & $\begin{array}{c}\text { Gastos do governo em } \\
\text { investimento }-\mathrm{R} \$\end{array}$ & $32.043 .655,61$ & $234.943 .211,60$ & $1.616 .466,38$ & $5.730 .289 .311,00$ \\
\hline & $\begin{array}{l}\text { Receita proveniente do fundo de } \\
\text { participação dos municípios - R\$ }\end{array}$ & $2.966 .890,25$ & $2.759 .806,81$ & 22,15 & $30.906 .879,00$ \\
\hline \multicolumn{6}{|c|}{ Forcas Centrífugas } \\
\hline $\begin{array}{l}\text { Variável } \\
\text { Teórica } \\
\end{array}$ & Proxy & & & & \\
\hline \multirow[t]{2}{*}{$\begin{array}{c}\text { Externalidades } \\
\text { negativas }\end{array}$} & $\begin{array}{c}\text { Densidade demográfica do } \\
\text { município medido em hab./Km² }\end{array}$ & 265,53 & $1.073,98$ & 3,93 & $11.632,46$ \\
\hline & $\begin{array}{l}\text { Taxa de homicídios por } 100.000 \\
\text { hab. }\end{array}$ & 21,62 & 18,36 & 2,17 & 108,54 \\
\hline \multirow[t]{2}{*}{ Tributação } & $\begin{array}{l}\text { Receitas municipais advindas da } \\
\text { arrecadação do imposto predial e } \\
\text { territorial urbano - R\$ }\end{array}$ & $4.447 .779,30$ & $53.323 .533,85$ & 0 & $1.328 .611 .120,00$ \\
\hline & $\begin{array}{l}\text { Receitas municipais advindas da } \\
\text { arrecadação do imposto sobre } \\
\text { serviços }-\mathrm{R} \$\end{array}$ & $4.213 .806,61$ & $66.772 .892,04$ & 0 & $1.673 .324 .008,00$ \\
\hline
\end{tabular}

As unidades espaciais adotadas consistem nos municípios paulistas, e a fonte fundamental é o Instituto Brasileiro de Geografia e Estatística (IBGE). Os dados sobre homicídios são do Sistema de Informações sobre Mortalidade do DATA-SUS e os dados sobre finanças municipais estão disponíveis no site da Secretaria do Tesouro Nacional. O período escolhido justifica-se pela disponibilidade de dados (ano 2000), pois alguns dados só são disponibilizados em determinados anos. Utilizou-se o PIB per capta como uma proxy para o crescimento econômico em um primeiro modelo e posteriormente estimou-se com os salários médios como proxy para o rendimento médio do trabalho. Vale lembrar que apenas a segunda variável é corroborada pelo modelo teórico apresentado.

\section{RESULTADOS E DISCUSSÃO}

Os resultados obtidos, para as regressões por mínimos quadrados ordinários para a equação (16), são apresentados nas Tabelas 1 e 2. Os resultados são divididos em três pares de regressões e são referentes às informações para os 645 municípios paulistas para o ano de 2000.

Devido a problemas de correlação, as variáveis: gasto do governo, investimento do governo e receitas advindas do FPM foram estimadas separadamente, como também 
as variáveis: área do município e densidade demográfica, que apresentam uma correlação, foram estimadas separadamente.

Em todas as regressões, foram realizados testes de multicolinearidade, heterocedasticidade, autocorrelação, normalidade nos resíduos e significância dos parâmetros. Os testes encontraram problemas na normalidade dos resíduos e na autocorrelação. Tal fato justifica-se, pois, uma vez identificada a existência de padrões espaciais de crescimento, ocorrem efeitos das externalidades espaciais. Estas podem se manifestar de dois modos: primeiro por meio da defasagem espacial, ou seja, à medida que uma cidade cresce, pressupõe-se que esta deva influenciar o crescimento de seus vizinhos; e, segundo, a influência espacial pode ser derivada de variáveis omitidas, que se manifestam por meio da autocorrelação dos resíduos. Esse problema, só é resolvido, utilizando modelos da econometria espacial, como o modelo espacial autoregressivo, o que foge do escopo do trabalho e pode ser implementado em trabalhos futuros. Os resultados são apresentados em seis regressões (Tabela 1).

Tabela 1: Crescimento Econômico nos municípios paulistas em 2000.

\begin{tabular}{|c|c|c|c|c|c|c|}
\hline \multicolumn{7}{|c|}{ Variável dependente: Log PIB per capta } \\
\hline & Equação & Equação & Equação & Equação & Equação & Equação \\
& $\mathbf{1}$ & $\mathbf{2}$ & $\mathbf{3}$ & $\mathbf{4}$ & $\mathbf{5}$ & $\mathbf{6}$ \\
\hline Intercepto & 7.814666 & 7.78729 & 7.8149 & 7.787764 & 7.805702 & 7.782729 \\
& 0.159828 & 0.156771 & 0.159837 & 0.156753 & 0.161597 & 0.160586 \\
Urbanização & 0.011716 & 0.0117 & 0.011705 & 0.011691 & 0.011635 & 0.011648 \\
& 0.001744 & 0.001746 & 0.001743 & 0.001746 & 0.001749 & 0.00175 \\
Área & $-6.65 \mathrm{E}-05$ & & $-6.58 \mathrm{E}-05$ & & $-6.58 \mathrm{E}-05$ & \\
& $7.83 \mathrm{E}-05$ & & $7.82 \mathrm{E}-05$ & & $7.99 \mathrm{E}-05$ &
\end{tabular}




\begin{tabular}{|c|c|c|c|c|c|c|}
\hline Distância & $\begin{array}{c}-4.43 \mathrm{E}-05 \\
1.53 \mathrm{E}-04\end{array}$ & $\begin{array}{c}-7.63 \mathrm{E}-05 \\
1.54 \mathrm{E}-04\end{array}$ & $\begin{array}{c}-4.41 \mathrm{E}-05 \\
1.53 \mathrm{E}-04\end{array}$ & $\begin{array}{c}-7.57 \mathrm{E}-05 \\
1.55 \mathrm{E}-04\end{array}$ & $\begin{array}{c}-3.27 \mathrm{E}-05 \\
1.63 \mathrm{E}-04\end{array}$ & $\begin{array}{c}-7.03 \mathrm{E}-05 \\
1.59 \mathrm{E}-04\end{array}$ \\
\hline Homicídios & $\begin{array}{c}-0.001733 \\
0.00156\end{array}$ & $\begin{array}{c}-0.001242 \\
0.001656\end{array}$ & $\begin{array}{c}-0.00173 \\
0.001563\end{array}$ & $\begin{array}{c}-0.001249 \\
0.001657\end{array}$ & $\begin{array}{c}-0.001762 \\
0.001612\end{array}$ & $\begin{array}{c}-0.001248 \\
0.001659\end{array}$ \\
\hline Densidade & & $\begin{array}{c}-5.81 \mathrm{E}-06 \\
2.31 \mathrm{E}-05\end{array}$ & & $\begin{array}{c}-5.51 \mathrm{E}-06 \\
2.30 \mathrm{E}-05\end{array}$ & & $\begin{array}{c}-5.68 \mathrm{E}-06 \\
2.46 \mathrm{E}-05\end{array}$ \\
\hline $\begin{array}{c}\text { Gastos do } \\
\text { governo }\end{array}$ & $\begin{array}{c}4.85 \mathrm{E}-11 \\
8.95 \mathrm{E}-11\end{array}$ & $\begin{array}{c}3.63 \mathrm{E}-11 \\
9.00 \mathrm{E}-11\end{array}$ & & & & \\
\hline $\begin{array}{c}\text { Investimento } \\
\text { do governo }\end{array}$ & & & $\begin{array}{c}4.85 \mathrm{E}-10 \\
9.56 \mathrm{E}-10\end{array}$ & $\begin{array}{c}3.51 \mathrm{E}-10 \\
9.59 \mathrm{E}-10\end{array}$ & & \\
\hline FPM & & & & & $\begin{array}{c}3.71 \mathrm{E}-09 \\
9.71 \mathrm{E}-09\end{array}$ & $\begin{array}{c}2.22 \mathrm{E}-09 \\
1.02 \mathrm{E}-08\end{array}$ \\
\hline IPTU & $\begin{array}{c}-1.25 \mathrm{E}-09 \\
4.46 \mathrm{E}-09\end{array}$ & $\begin{array}{c}-1.58 \mathrm{E}-09 \\
5.61 \mathrm{E}-09\end{array}$ & $\begin{array}{c}-1.11 \mathrm{E}-09 \\
5.61 \mathrm{E}-09\end{array}$ & $\begin{array}{c}-1.47 \mathrm{E}-09 \\
5.61 \mathrm{E}-09\end{array}$ & $\begin{array}{c}-1.20 \mathrm{E}-09 \\
5.61 \mathrm{E}-09\end{array}$ & $\begin{array}{c}-1.54 \mathrm{E}-09 \\
5.61 \mathrm{E}-09\end{array}$ \\
\hline ISS & $\begin{array}{c}1.62 \mathrm{E}-09 \\
5.61 \mathrm{E}-09\end{array}$ & $\begin{array}{c}1.87 \mathrm{E}-09 \\
4.46 \mathrm{E}-09\end{array}$ & $\begin{array}{c}1.50 \mathrm{E}-09 \\
4.46 \mathrm{E}-09\end{array}$ & $\begin{array}{c}1.78 \mathrm{E}-09 \\
4.46 \mathrm{E}-09\end{array}$ & $\begin{array}{c}1.57 \mathrm{E}-09 \\
4.46 \mathrm{E}-09\end{array}$ & $\begin{array}{c}1.84 \mathrm{E}-09 \\
4.46 \mathrm{E}-09\end{array}$ \\
\hline $\mathbf{R}^{2}$ & 0.132619 & 0.131029 & 0.13253 & 0.13096 & 0.132262 & 0.130752 \\
\hline
\end{tabular}

Fonte: estimativas da pesquisa

Nota: Erro padrão abaixo dos coeficientes calculados.

Uma vez que, o enfoque principal deste artigo, diz respeito ao papel do setor público no crescimento econômico das cidades do estado de São Paulo, as variáveis que dizem respeito à política fiscal foram analisadas mais criteriosamente. As outras variáveis são variáveis de controle no modelo.

As estimativas estão de acordo com a teoria, com exceção dos resultados para os efeitos da área (tamanho da cidade em $\mathrm{km}^{2}$ ) que são negativos, e para os efeitos do ISS (Imposto sobre Serviços), que são positivos. O sinal invertido, na variável área, pode se dever ao fato do Brasil não apresentar problemas de escassez de terras (OLIVEIRA et $a l$, 2009). Portanto, uma cidade com mais terras disponíveis, não necessariamente apresenta um maior crescimento econômico. Já o impacto positivo do ISS pode acontecer, indicando aquecimento da atividade econômica e maior geração de receitas, o que permite um governo local mais presente em termos de gastos. Além disso, partese do pressuposto que o imposto apresenta relação causal com a atividade econômica, relação que pode ser inversa.

Os resultados obtidos, para o grau de urbanização dos municípios, pode ser um indicativo do diferente estágio de desenvolvimento, vivido pela economia paulista. No modelo estimado, um município mais urbanizado possui um potencial de crescimento 
maior. A distância à capital estadual manteve sua relação inversa com o crescimento municipal, ou seja, quanto mais distante da capital, ceteris paribus, menor tende a ser a taxa de crescimento média do município.

Foi realizado o mesmo procedimento, para a variável dependente salários médios, e foi alterada também a proxy de externalidade positiva, substituída pela média de anos de estudo, para um maior alinhamento com o modelo proposto por Glaeser (2000). Também foram incluídas variáveis dummies. Tal inclusão é um controle importante, tendo o objetivo de captar as diferenças regionais de crescimento econômico. Por ter o maior crescimento econômico e ser a região em que se localiza a capital do estado de São Paulo, escolheu-se a região sul como região de comparação. Os resultados encontram-se na Tabela 2.

A interpretação de alguns parâmetros do modelo exige algum cuidado. A variável homicídios impacta positivamente o crescimento econômico. Modelos econométricos devem ser analisados com os devidos cuidados, e o modelo por si só não diz tudo. O sinal positivo desta variável pode ser coerente, uma vez que as regiões de maior aglomeração de pessoas apresentam indicadores mais elevados de criminalidade em geral.

Tabela 2: Salários Médios nos municípios paulistas em 2000

\begin{tabular}{|c|c|c|c|c|c|c|}
\hline \multicolumn{7}{|c|}{ Variável dependente: Log Salários Médios } \\
\hline & $\begin{array}{c}\text { Equação } \\
1\end{array}$ & $\begin{array}{c}\text { Equação } \\
2\end{array}$ & \begin{tabular}{|c|} 
Equação \\
$\mathbf{3}$ \\
\end{tabular} & $\begin{array}{c}\text { Equação } \\
4\end{array}$ & \begin{tabular}{|c|} 
Equação \\
5
\end{tabular} & $\begin{array}{c}\text { Equação } \\
6\end{array}$ \\
\hline Intercepto & $\begin{array}{c}7.814666 \\
0.159828\end{array}$ & $\begin{array}{c}7.78729 \\
0.156771\end{array}$ & & $\begin{array}{c}7.787764 \\
0.156753\end{array}$ & $\begin{array}{c}7.805702 \\
0.161597\end{array}$ & $\begin{array}{c}7.782729 \\
0.160586\end{array}$ \\
\hline Estudo & $\begin{array}{c}0.011716 \\
0.001744\end{array}$ & $\begin{array}{l}0.0117 \\
0.001746\end{array}$ & $\begin{array}{c}0.011705 \\
0.001743\end{array}$ & $\begin{array}{c}0.011691 \\
0.001746\end{array}$ & $\begin{array}{c}0.011635 \\
0.001749\end{array}$ & $\begin{array}{c}0.011648 \\
0.00175\end{array}$ \\
\hline Área & $\begin{array}{c}-6.65 \mathrm{E}-05 \\
7.83 \mathrm{E}-05\end{array}$ & & $\begin{array}{c}-6.58 \mathrm{E}-05 \\
7.82 \mathrm{E}-05\end{array}$ & & $\begin{array}{c}-6.58 \mathrm{E}-05 \\
7.99 \mathrm{E}-05\end{array}$ & \\
\hline Distância & $-4.43 E-05$ & $-7.63 \mathrm{E}-05$ & $-4.41 E-05$ & $-7.57 \mathrm{E}-05$ & $-3.27 \mathrm{E}-05$ & $-7.03 \mathrm{E}-05$ \\
\hline
\end{tabular}




\begin{tabular}{|c|c|c|c|c|c|c|}
\hline & $1.53 \mathrm{E}-04$ & $1.54 \mathrm{E}-04$ & $1.53 \mathrm{E}-04$ & $1.55 \mathrm{E}-04$ & $1.63 \mathrm{E}-04$ & $1.59 \mathrm{E}-04$ \\
\hline Homicídios & $\begin{array}{c}-0.001733 \\
0.00156\end{array}$ & $\begin{array}{c}-0.001242 \\
0.001656\end{array}$ & $\begin{array}{c}-0.00173 \\
0.001563\end{array}$ & $\begin{array}{c}-0.001249 \\
0.001657\end{array}$ & $\begin{array}{c}-0.001762 \\
0.001612\end{array}$ & $\begin{array}{c}-0.001248 \\
0.001659\end{array}$ \\
\hline Densidade & & $\begin{array}{c}-5.81 \mathrm{E}-06 \\
2.31 \mathrm{E}-05\end{array}$ & & $\begin{array}{c}-5.51 \mathrm{E}-06 \\
2.30 \mathrm{E}-05\end{array}$ & & $\begin{array}{c}-5.68 \mathrm{E}-06 \\
2.46 \mathrm{E}-05\end{array}$ \\
\hline $\begin{array}{c}\text { Gastos do } \\
\text { governo }\end{array}$ & $\begin{array}{c}4.85 \mathrm{E}-11 \\
8.95 \mathrm{E}-11\end{array}$ & $\begin{array}{c}3.63 \mathrm{E}-11 \\
9.00 \mathrm{E}-11\end{array}$ & & & & \\
\hline $\begin{array}{l}\text { Investimento } \\
\text { do governo }\end{array}$ & & & $\begin{array}{c}4.85 \mathrm{E}-10 \\
9.56 \mathrm{E}-10\end{array}$ & $\begin{array}{c}3.51 \mathrm{E}-10 \\
9.59 \mathrm{E}-10\end{array}$ & & \\
\hline FPM & & & & & $\begin{array}{c}3.71 \mathrm{E}-09 \\
9.71 \mathrm{E}-09\end{array}$ & $\begin{array}{c}2.22 \mathrm{E}-09 \\
1.02 \mathrm{E}-08\end{array}$ \\
\hline IPTU & $\begin{array}{c}-1.25 \mathrm{E}-09 \\
4.46 \mathrm{E}-09\end{array}$ & $\begin{array}{c}-1.58 \mathrm{E}-09 \\
5.61 \mathrm{E}-09\end{array}$ & $\begin{array}{c}-1.11 \mathrm{E}-09 \\
5.61 \mathrm{E}-09\end{array}$ & $\begin{array}{c}-1.47 \mathrm{E}-09 \\
5.61 \mathrm{E}-09\end{array}$ & $\begin{array}{c}-1.20 \mathrm{E}-09 \\
5.61 \mathrm{E}-09\end{array}$ & $\begin{array}{c}-1.54 \mathrm{E}-09 \\
5.61 \mathrm{E}-09\end{array}$ \\
\hline ISS & $\begin{array}{c}1.62 \mathrm{E}-09 \\
5.61 \mathrm{E}-09\end{array}$ & $\begin{array}{c}1.87 \mathrm{E}-09 \\
4.46 \mathrm{E}-09\end{array}$ & $\begin{array}{c}1.50 \mathrm{E}-09 \\
4.46 \mathrm{E}-09\end{array}$ & $\begin{array}{c}1.78 \mathrm{E}-09 \\
4.46 \mathrm{E}-09\end{array}$ & $\begin{array}{c}1.57 \mathrm{E}-09 \\
4.46 \mathrm{E}-09\end{array}$ & $\begin{array}{c}1.84 \mathrm{E}-09 \\
4.46 \mathrm{E}-09\end{array}$ \\
\hline $\mathbf{R}^{2}$ & 0.132619 & 0.131029 & 0.13253 & 0.13096 & 0.132262 & 0.130752 \\
\hline
\end{tabular}

Fonte: Estimativas da pesquisa

Nota: Erro padrão abaixo dos coeficientes calculados

No caso da densidade demográfica, conforme apresentado pela NGE, locais que apresentam uma elevada densidade demográfica são propícios para o surgimento de criminalidade, favelas, poluição, que formam forças inibidoras para o crescimento. Por outro lado, uma maior densidade demográfica, pode levar a um maior número de indivíduos que se dedicam ao desenvolvimento de novas tecnologias, contribuindo para a proliferação das mesmas dentro da sociedade, o que provoca um efeito positivo para o crescimento econômico e justificaria o sinal positivo da variável.

Outro parâmetro que obteve sinal contraditório foi a variável representativa dos anos médios de estudo, que apresentou sinal negativo e significativo estatisticamente, indicando que, quanto maior o nível educacional inicial da população, menos o município tende a crescer. Isso pode acontecer, pois a escolaridade média, de pessoas com 25 anos ou mais, não necessariamente é uma variável representativa do nível de capital humano de uma região.

Feitas as considerações a respeito das variáveis de controle, discutem-se os efeitos da política fiscal local, no crescimento econômico das cidades paulistas. Os resultados mostram um efeito positivo da variável gastos do governo. Isso significa que 
o resultado está de acordo com o modelo teórico, ou seja, a política físcal afeta positivamente o crescimento econômico das cidades do estado de São Paulo.

O modelo também mostra que o investimento público é fundamental para o crescimento econômico. Este tipo de gasto, certamente afeta a qualidade de vida e a produtividade dos indivíduos, gerando externalidades que impactam positivamente o crescimento econômico dos municípios. Além disso, o investimento público local pode ser um dos determinantes na atratividade de investimentos privados, o que também induz o crescimento econômico.

No caso do Fundo de Participação dos Municípios (FPM), um mecanismo de redistribuição fiscal com a finalidade de reduzir as desigualdades regionais, cumpre-se o referido papel, segundo o resultado das estimações. O efeito positivo do FPM mostra que esse tipo de gasto gera benefícios locais, além disso, vale ressaltar, que muitos municípios dependem das transferências constitucionais, para financiamento de seus gastos.

No âmbito dos tributos locais, que foram incluídos no modelo econométrico, os resultados do IPTU são os previstos no modelo teórico proposto. Neste caso, a tributação atua como uma força centrífuga e, portanto, afeta negativamente o crescimento econômico das cidades. Vale salientar, que a cobrança deste imposto sobre o patrimônio, aumenta os custos da terra, o que, conforme a NGE, é um importante insumo do processo de produção.

\section{CONCLUSÕES}

O principal objetivo deste trabalho é o de investigar o papel da política fiscal local, no crescimento econômico dos municípios do estado de São Paulo. Para este fím, a pesquisa apresenta um modelo espacial de crescimento econômico com governo, que incorpora contribuições das teorias do crescimento endógeno, e da nova geografia econômica. Com o propósito de descrever os efeitos da ação de política fiscal, elaborada pelas prefeituras dos municípios paulistas, utilizou-se o modelo econométrico, que evidencia tais efeitos e verificou-se de maneira consistente, que as ações do governo apresentam forte impacto, sobre o crescimento econômico dos 645 municípios estudados, conforme as hipóteses iniciais levantadas no presente trabalho.

Este resultado mostra que gastos públicos podem ajudar a equilibrar a distribuição espacial das atividades, e afetar positivamente o crescimento econômico 
dos municípios. Nota-se, que os gastos servem como fonte de uma melhor qualidade de vida, possibilitando um padrão mais elevado da produtividade dos diversos setores econômicos, encontrados no estado de São Paulo. Além disso, cabe destacar que a política fiscal deve ser bem elaborada, e assim, as prefeituras conseguirão evitar os efeitos nocivos que o IPTU promove sobre o crescimento. Portanto, o papel do governo no crescimento das cidades é indiscutível. A partir dos resultados foi possível observar que uma política fiscal adequada pode atrair investimentos para locais mais remotos, superando as forças de aglomeração, explicadas pela NGE.

Uma das principais dificuldades, para a realização do trabalho, foi a falta de dados de qualidade, que permitissem a utilização de um período maior de tempo e de métodos, tais como o da regressão em painel, de modo a se obterem resultados mais robustos. Com relação aos desafios teóricos e empíricos, comumente enfrentados pelos estudiosos do crescimento, planeja-se avançar na questão futuramente, por meio da combinação de ferramentas teóricas e econométricas, que possibilitam melhores resultados.

\section{REFERÊNCIAS BIBLIOGRÁFICAS}

ASSIS, L. C.; DIAS, J. Política fiscal, nível tecnológico e crescimento econômico no Brasil: teoria e evidência empírica. In: Anais do XXXII Encontro Nacional de Economia. João Pessoa, 2004.

AZZONI, C.R. Equilíbrio, progresso técnico e desigualdades regionais no processo de desenvolvimento econômico. Análise Econômica. Porto Alegre, ano XI, n 19 março/1993.

BARRO, R. J. Government spending in a simple model of endogenous growth. Journal of Political Economy, v. 98, p. 103-125, Oct. 1990.

CÂNDIDO Jr, J. O. Texto para Discussão do IPEA, n 781 . Os Gastos Públicos são Produtivos? Brasília, 2001.

CHAGAS, A. L. S. TONETO JR, R. Fatores determinantes do crescimento local evidências a partir de dados dos municípios brasileiros para o período de 1980 a 1991. Pesquisa e Planejamento Econômico. V.33, n 2, 349-385, 2003.

COSTA, R. F.; LIMA, F. S.; SILVA, D. O. Política fiscal local e crescimento econômico: Um escudo em painel para os municípios nordestinos. Revista econômica do nordeste, vol. 6, 2007. 
COSTA, R. F. R.; LIMA, F. S. O impacto da política fiscal local sobre o crescimento econômico: um estudo em cross-section para os municípios potiguares. In: Anais do XIII Encontro Regional de Economia. Fortaleza - CE, 2008.

FUNDAÇÃO SISTEMA ESTADUAL DE ANALISE DE DADOS - SEADE. Boletim SEADE. São Paulo, 2006. Disponível em: http://www.seade.gov.br/. Acesso em: 22/03/2011.

FUNDAÇÃO SISTEMA ESTADUAL DE ANALISE DE DADOS - SEADE. Boletim SEADE. São Paulo, 2010. Disponível em: http://www.seade.gov.br/. Acesso em: 22/03/2011.

Giambiagi, F. AlÉM, A. C. Finanças Públicas: Teoria e Prática no Brasil. $3^{\mathrm{a}}$ Ed. Rio de Janeiro: Editora Campus. 2008. 496p.

GLAESER, E.L.; SCHEINKMAN, J. A.; SHLEIFER, A."Economic Growth in a Crosssection of Cities” Journal of Monetary Economics. 36(1), p. 117-143, 1995.

"The new economics of urban and regional growth" in: The Oxford Handbook of Economic Geography, Gordon L. Clark, Maryann P. Feldman, and Meric S. Gertler (eds). Oxford University Press: Oxford, 2000.

GONÇALVES, J.S. RESENDE, J.V. Transformações econômico-sociais e a evolução da Pesquisa Agropecuária Paulista. Agricultura em São Paulo. 40(2): 1-35, 1993

HENDERSON, J.V. 1986. Economic Theory and the Cities. New York: Academic Press.

HERRERA, S.; BLANCO, F. The quality of fiscal adjustment and the long run growth impact of fiscal policy in brazil. Mimeo. 2004.

HOOVER, E.M., GIARRANTI, F. 1984. An introduction to regional economics, 3rd ed. New York: Alfred A. Knopf.

INSTITUTO DE PESQUISA ECONÔMICA APLICADA - IPEA. Ipeadata. 2011. Disponível em http://www.ipeadata.gov.br/. Acesso em março de 2011.

KWEKA, J.P.; MORRISSEY, O. Government Spending and Economic Growth in Tanzania, 1965-1996. Texto para discussão, 00/6, School of Economics, University of Nottingham, 2000.

KRUGMAN, P. Increasing Returns and Economic Geography. Journal of Political Economy, vol 99, n 3, jun 1991, pp. 483-499.

LUCAS, R.E. On the mechanics of economic development. Journal of Monetary Economics, v. 22, n. 1, p. 3-42, July 1988.

MUSGRAVE, R. A. The Theory of Public Finance. New York: McGraw-Hill, 1959. 
OLIVEIRA, C. Crescimento Econômico das Cidades Nordestinas: Um enfoque da Nova Geografia Econômica. Revista Econômica do Nordeste, vol. 3, 2004.

OLIVEIRA, C. A. Política fiscal local e o crescimento econômico dos municípios gaúchos (1996 - 2001) In: Anais do III Encontro de Economia Gaúcho. Porto Alegre, 2006.

OLIVEIRA, C.A, MARQUES Jr, L. dos S.. JACINTO, P.A. Política fiscal local e seu papel no Crescimento Econômico - uma evidência empírica para o Brasil. Revista EconomiA, v. 10, n.1, p. 49-68, jan-abr 2009.

REZENDE, F. Finanças Públicas. 2 ed. São Paulo: Atlas. 2007, 382p.

ROCHA, F.; GIUBERTI, A. C. Composição do gasto público e crescimento econômico: um estudo em painel para os estados brasileiros. In: Anais do XXXIII Encontro Nacional de Economia. Natal, 2005.

ROMER, P. M. Increasing returns and long run growth. Journal of Political Economy, v. 94, n. 5, p. 1002-37, Out. 1986.

RUIZ, R. M. Políticas regionais na nova geografia econômica. Ministério da Integração, Projeto: Diretrizes para formulação de políticas de desenvolvimento regional e de ordenação do território brasileiro. Belo Horizonte - MG, 2004.

SECRETARIA DE ECONOMIA E PLANEJAMENTO. Desenvolvimento regional. São Paulo, 2007. Disponível em: http://www.planejamento.sp.gov.br/index.aspx. Acesso em: 09/03/2011.

SOLOW, R.M. A contribution to the theory of economic growth. Quaterly Journal of Economics, v. 70, n. 1, p. 65-94, Feb. 1956.

SWAN, T.W. Economic growth and capital accumulation. Economic Record, v. 32, p. 334-361, Nov. 1956.

VON THÜNEN, J.H. The Isolated State. Hamburg: Perthes, 1826. English translation. Oxford: Pergamon, 1966. 